\title{
Association of signalment parameters with activity of pet dogs*
}

\author{
Kathryn E. Michel ${ }^{1 \dagger}$ and Dorothy C. Brown ${ }^{2}$ \\ ${ }^{1}$ Department of Clinical Studies - Philadelphia, School of Veterinary Medicine, University of Pennsylvania, 3900 Delancey Street, Philadelphia, \\ PA 19104-6010, USA \\ ${ }^{2}$ Department of Clinical Studies - Philadelphia, School of Veterinary Medicine, 3900 Delancey Street, Philadelphia, PA 19104-6010, USA, \\ and Center for Clinical Epidemiology and Biostatistics, School of Medicine, University of Pennsylvania, 423 Guardian Drive, Philadelphia, \\ PA 19104-6021, USA
}

(Received 23 January 2014 - Final revision received 15 May 2014 - Accepted 5 June 2014)

Journal of Nutritional Science (2014), vol. 3, e28, page 1 of 4

doi:10.1017/jns.2014.49

Abstract

Activity monitors are increasingly being used to quantify the activity of pet dogs. The aim of the present study was to investigate associations between signalment and activity of free-living pet dogs. Healthy pet dogs were recruited to wear an Actical activity monitor on their collars continuously for 2 weeks in their home environment. At least fifteen dogs were enrolled in each of the following weight ranges: $<10,10-20,21-30,31-40,>40 \mathrm{~kg}$ and their age, sex and reproductive status recorded. Each dog's intensity of activity for each minute of recording was classified using the total counts for that minute and our pre-established cut-points. The percentage of time dogs spent in sedentary, light or moderate/vigorous activity each day was calculated. Median total daily activity counts and median percentages of time dogs spent in activities of differing intensity were used for the analysis. Associations between signalment characteristics and activity parameters were evaluated with the Mann-Whitney test, the Kruskal-Wallis test and Spearman rank correlations. Ninety-eight dogs were enrolled with $\geq 17 \mathrm{dogs}$ in each weight category. Time that dogs were sedentary correlated positively with age $(r 0.50$, uncorrected $P<0 \cdot 001)$, while the median total daily activity count $(r-0 \cdot 47$, uncorrected $P<0 \cdot 001)$, time spent in light $(r-0 \cdot 46$, uncorrected $P<0.001)$ and more vigorous activity $(r-0.50$, uncorrected $P<0.001)$ were negatively associated with age. No other significant associations between signalment and activity parameters were found. The lack of differences in activity across weight categories may reflect the impact of lifestyle negating any potential behavioural differences across breeds.

Key words: Canine nutrition: Dogs: Physical activity: Accelerometry

Canine daily energy requirements have been principally investigated using data collected from dogs kennelled in a controlled environment ${ }^{(1)}$. Use of kennelled dogs allows for control of variables such as diet, energy intake and body weight. However, the conditions under which kennelled dogs are housed differ in a number of respects from those of pet dogs. For example, the housing of pet dogs can vary substantially among individual households and the kinds of activities in which pet dogs participate are likely to be much more variable and uncontrolled in comparison to dogs kennelled in a controlled environment and are likely influenced by the activities of their owners. There is considerable variation among individuals in the daily maintenance energy requirement of healthy adult dogs. This is due to differences in metabolism, body composition and lifestyle. It may also be influenced by signalment factors including sex, reproductive status, age and breed.

We and others have been investigating an accelerometerbased device that can continuously measure the intensity, frequency and duration of movement for extended periods for use in monitoring activity in pet dogs (Actical, Mini Mitter Inc ${ }^{(2-9)}$. We have previously reported that there is no significant impact of signalment or body conformation on the average activity counts delivered by this monitor when the activity

†Corresponding author: Dr Kathryn E. Michel, fax +1 215573 6050, email michel@vet.upenn.edu

* This article was published as part of the WALTHAM International Nutritional Sciences Symposium Proceedings 2013. 
is well controlled and all dogs perform the same movements ${ }^{(2)}$. Furthermore, we found that a 7-d data collection period provides relatively stable estimates of activity in dogs while including the days with the highest potential for changes in activity to occur (weekends) ${ }^{(3)}$. We have also shown that an activity monitor can be used to differentiate the intensity of activity of pet dogs through the establishment of activity count cut-points that can be used to discriminate among sedentary, light and more vigorous activities ${ }^{(4)}$. The ability to quantify activity level in free-living pet dogs and the factors that influence activity could lead to a better understanding of the energy expenditure of pet dogs and the ability to more accurately predict daily energy needs. The aim of the present study was to use accelerometry to investigate associations between signalment and activity of free-living pet dogs.

\section{Materials and methods}

\section{Dogs}

The protocol used in this investigation was in accordance with University of Pennsylvania and United States guidelines for the care and use of animals and was fully approved by the University of Pennsylvania Institutional Animal Care and Use Committee. Written informed consent was obtained from owners of all participating dogs prior to enrolment. Pet dogs belonging to the staff and students of the School of Veterinary Medicine of the University of Pennsylvania were recruited to enrol in this study. For inclusion, the dog had to be in good health based upon medical history and physical examination and in optimal body condition (defined as greater than or equal to a body condition score of $4 / 9$ but less than a body condition score of 6/9) and there could not be any anticipated changes in the household's typical schedule during the data collection period (e.g. vacation, boarding, etc.). If malfunction or removal of the activity monitor during the study period occurred, the dog was removed from study. To ensure inclusion of a diverse cohort, at least fifteen dogs were recruited in each of the following five weight ranges: $<10$, $10-20,21-30,31-40,>40 \mathrm{~kg}$; however, a power calculation was not performed. In each case, signalment data were recorded including age (in years rounded to the nearest half year), sex, reproductive status and breed.

\section{Experimental design}

In all cases, the Actical Activity Monitor, an omnidirectional accelerometer-based device that measures continuously the intensity, frequency and duration of movement, was used. A detailed description of the monitor and how it works is reported elsewhere ${ }^{(5)}$. The watch-sized monitor was attached to the collar of each dog and positioned ventrally on the neck. The dog's weight was determined using a single calibrated walk-on scale (Arlyn Scales) and recorded. The dog wore the monitor continuously for 2 weeks in its routine home environment at the end of which time it returned to be weighed and for collection of the monitor. The accelerometer data epoch was set at 1 min to permit data collection over an extended period of time.

\section{Data analysis}

Each dog's intensity of activity for each minute of recording was classified using the total counts for that minute and our pre-established cut-points ${ }^{(2,4)}$. The percentage of time dogs spent in sedentary, light or moderate/vigorous activity each day was calculated. Median total daily activity counts and median percentages of time dogs spent in activities of differing intensity were used for the analysis. Because activity data were not normally distributed, nonparametric methods of analysis were used. Associations between sex or reproductive status and activity parameters were evaluated with the MannWhitney test, between weight category and activity parameters with the Kruskal-Wallis test, and between age, weight and activity parameters with Spearman rank correlation. All tests were two-sided. As twenty comparisons were made between the four activity parameters and five signalment variables, a multiple comparisons correction was applied to the uncorrected overall critical $P$ value of $0 \cdot 05$. The corrected overall critical $P$ value was $0 \cdot 003$. All analyses were performed using commercially available software (Stata version 8, StataCorp, College Station, Tex, USA).

\section{Results}

One hundred dogs were recruited and ninety-eight dogs, ranging in age from 1 to 14 years (median age $=3$ years), were included in the analysis with at least seventeen dogs in each weight category $(<10 \mathrm{~kg}, n 21 ; 10-20 \mathrm{~kg}, n 20 ; 21-30 \mathrm{~kg}$, $n$ 21; 31-40 kg, n 18; >40 kg, n 17). No dogs dropped out of the study once they were recruited. Two of the recruited dogs were excluded from the analysis, one because the collar with the affixed monitor came off of the dog for an extended period during the 2-week-monitoring period and one because the dog's weight was not recorded at the time of enrolment into the study. There were dogs representing thirty-three different breeds as well as thirty-eight mixed breed dogs included; however, no single breed was represented by more than four individuals. Table 1 shows the data for the associations among activity parameters and sex or reproductive status. Table 2 shows the data for the associations among activity parameters and weight categories. No significant associations were found. The median percentage time that dogs were sedentary was positively correlated with age $(r 0.50$, uncorrected $P<0.001)$, while the median total daily activity count $(r-0 \cdot 47$, uncorrected $P<0 \cdot 001)$, median percentage of time spent in light $(r-0.46$, uncorrected $P<0.001)$ and moderate/vigorous activity $(r-0 \cdot 50$, uncorrected $P<0 \cdot 001)$ were negatively associated with age. No other significant associations between signalment and activity parameters were found. 

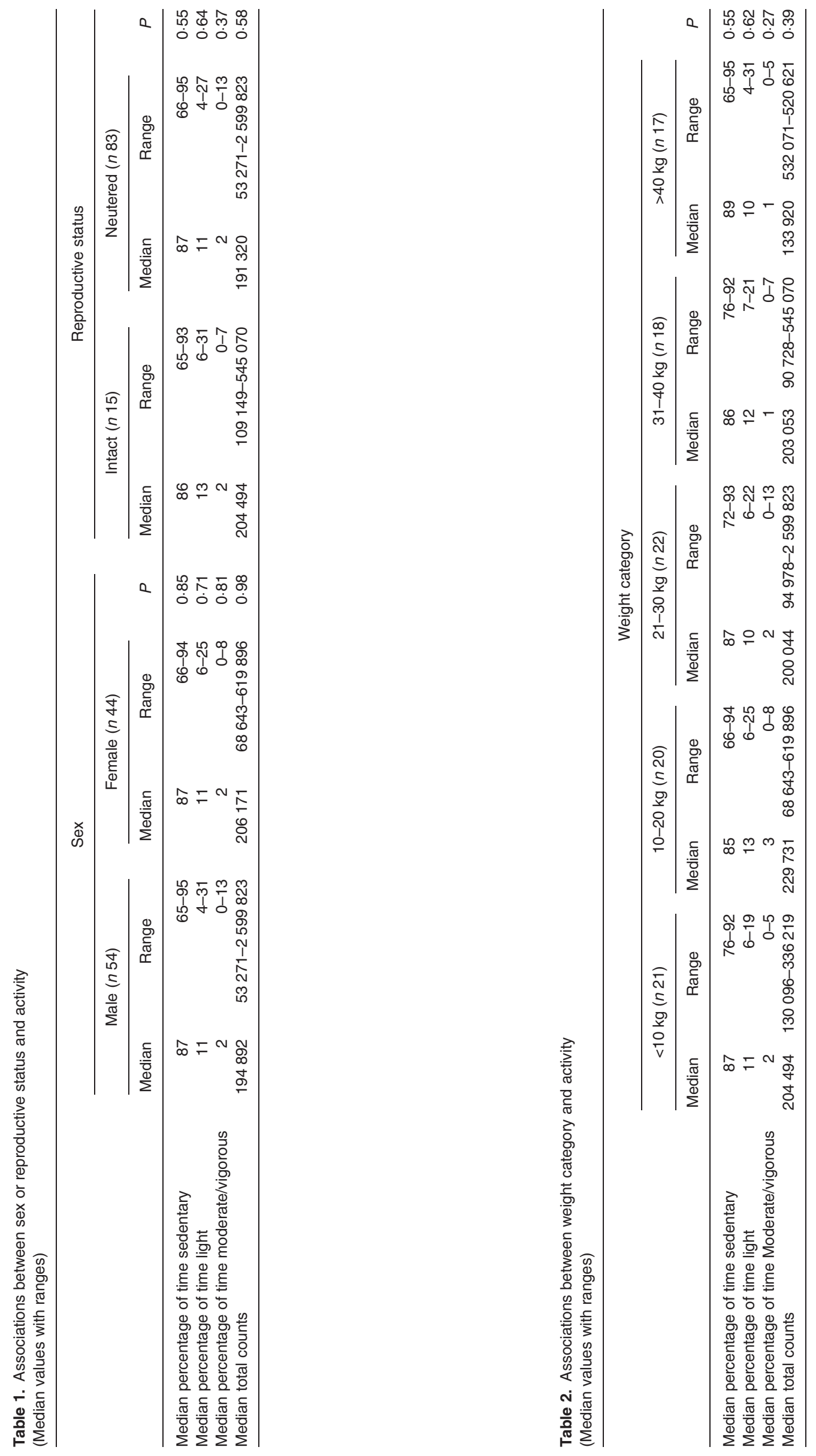


\section{Discussion}

Using acclerometry to monitor activity of pet dogs, we found that the amount of time that dogs were sedentary correlated positively with age while the median total daily activity count and time spent in light and more vigorous activity were negatively associated with age. No other significant associations between signalment and activity parameters were found.

The daily energy requirement of kennelled dogs can be approximated under controlled conditions with reasonable accuracy. The degree that the predictive equations for canine daily energy requirement that are based on data from kennelled dogs reflect the requirements of pet dogs in a home environment is open to question as there has been little published information regarding the pet population ${ }^{(1)}$. Physical activity, along with the metabolic demands of basic life processes and energy expended as necessary for thermoregulation and assimilation of nutrients from the diet, accounts for the daily maintenance energy requirement of a healthy adult dog and can show considerable variation among individuals. In recent years, accelerometry has shown promise as a means of quantifying physical activity in $\operatorname{dogs}^{(2-9)}$. In the present investigation, our aim was to look for associations between signalment and activity parameters in free-living pet dogs. If a strong association between activity and one or more aspects of signalment could be found, this information would be helpful for providing more accurate feeding recommendations for pet dogs.

In the present investigation aging was associated with a greater percentage of time sedentary and a smaller percentage of time spent in light or moderate/vigorous activities. These findings are consistent with those reported from an investigation using accelerometry in kennelled beagle dogs. In that study, higher total activity counts were recorded in young (1-4 years) $v$. older (9-14 years) dogs ${ }^{(6)}$.

We did not find any other statistically significant associations between signalment and activity parameters in this population. Specifically with regard to body weight, there were no significant associations with activity regardless of whether the data were analysed by weight category or for the entire data set of all ninety-eight dogs. This finding is contrary to the perception that certain dog breeds are more or less active. There are limitations to the present study. Although a wide range of body weights and breeds were included in our population, the sample size was not based on a power calculation and no single breed was represented by more than four individuals. It is possible that the lack of differences in activity across weight categories may reflect the impact of lifestyle negating potential behavioural differences across breeds (although, in this investigation we did not attempt to gather and correlate specific information about how the participating dogs were housed or exercised due to the extended interval of time that data were collected over).

In summary using acclerometry in a cohort of pet dogs, of the signalment parameters studied, only age was found to be associated with activity. The present study should be instrumental in insuring design for sufficient power in the future investigations encompassing greater representation of popular breeds. Inclusion of characteristics of the participant's lifestyle to allow investigation of the impact of environment and husbandry is also warranted.

\section{Acknowledgements}

The present research was supported by the Nestle Purina PetCare Company and the National Institutes of Health (D. B., grant No. 1-K08-DA-017720-02). Neither the Nestle Purina PetCare Company nor the National Institutes of Health had any role in the design, analysis or writing of the present paper. One of the authors, K. E. M. serves on a scientific advisory board for the Nestle Purina PetCare Co. D. C. B. has no conflicts of interest to report. K. E. M. was principally responsible for formulating the research question, the study design, data collection and the manuscript preparation and contributed to the data analysis. D. C. B. was principally responsible for the data analysis and contributed to the study design, data collection and manuscript preparation.

This paper was published as part of the WALTHAM International Nutritional Sciences Symposium Proceedings 2013, publication of which was supported by an unrestricted educational grant from Mars Incorporated. The papers included in these proceedings were invited by the Guest Editor and have undergone the standard journal formal review process. They may be cited.

\section{References}

1. Ad Hoc Committee on Dog and Cat Nutrition (2006) Energy. In Nutrient Requirements of Dogs and Cats, pp. 28-48. Washington, DC: National Academies Press.

2. Brown DC, Michel KE, Love M, et al. (2010) The effect of signalment and body conformation on activity monitoring in companion dogs. Am J Vet Res 71, 322-325.

3. Dow C, Michel KE, Love M, et al. (2009) Investigation of an optimal sampling interval for activity monitoring in companion dogs. Am J Vet Res 70, 444-448.

4. Michel KE \& Brown DC (2011) Determination and application of cut points for accelerometer-based activity counts of activities with differing intensity in pet dogs. Am J Vet Res 72, 866-870.

5. Hansen BD, Lascelles BDX, Keene BW, et al. (2007) Evaluation of an accelerometer for at-home monitoring of spontaneous activity in dogs. Am J Vet Res 68, 468-475.

6. Siwak CT, Tapp PD, Zicker SC, et al. (2003) Locomotor activity rhythms in dogs vary with age and cognitive status. Behav Neurosci 117, 813-824.

7. Yam PS, Penpraze V, Young D, et al. (2011) Validity, practical utility and reliability of Actigraph accelerometry for the measurement of habitual physical activity of dogs. J Sm Anim Pract 52, 86-92.

8. Wrigglesworth DJ, Mort ES, Upton SL, et al. (2011) Accuracy of the use of triaxial accelerometry for measuring daily activity as a predictor of daily maintenance energy requirement in healthy adult Labrador Retrievers. Am J Vet Res 72, 1151-1155.

9. Morrison R, Penpraze V, Beber A, et al. (2013) Associations between obesity and physical activity in dogs: a preliminary investigation. $J \mathrm{Sm}$ Anim Pract 54 570-574. 